\title{
Gastroesophageal Reflux in Neurologically Impaired Children: What Are the Risk Factors?
}

\author{
Seung Kim, Hong Koh, and Joon Soo Lee \\ Department of Pediatrics, Severance Hospital, Yonsei University College of Medicine, Seoul, Korea
}

Background/Aims: Neurologically impaired patients frequently suffer from gastrointestinal tract problems, such as gastroesophageal reflux disease (GERD). In this study, we aimed to define the risk factors for GERD in neurologically impaired children. Methods: From May 2006 to March 2014, 101 neurologically impaired children who received 24-hour esophageal pH monitoring at Severance Children's Hospital were enrolled in the study. The esophageal $\mathrm{pH}$ finding and the clinical characteristics of the patients were analyzed. Results: The reflux index was higher in patients with abnormal electroencephalography (EEG) results than in those with normal EEG results $(p=0.027)$. Mitochondrial disease was associated with a higher reflux index than were epileptic disorders or cerebral palsy $(p=0.009)$. Patient gender, feeding method, scoliosis, tracheostomy, and baclofen use did not lead to statistical differences in reflux index. Age of onset of neurological impairment was inversely correlated with DeMeester score and reflux index. Age at the time of examination, the duration of the disease, and the number of antiepileptic drugs were not correlated with GER severity. Conclusions: Early-onset neurological impairment, abnormal EEG results, and mitochondrial disease are risk factors for severe GERD. (Gut Liver 2017;11:232-236)

Key Words: Gastroesophageal reflux; Esophageal pH monitoring; Child

\section{INTRODUCTION}

As the central nervous system controls the enteric nervous system, neurologically impaired patients (NIP) frequently suffer from gastrointestinal tract dysfunction. ${ }^{1}$ Del Giudice et al. ${ }^{2}$ reported that $92 \%$ of children with cerebral palsy had clinically significant gastrointestinal symptoms: gastroesophageal reflux,
77\%; swallowing difficulties, 60\%; chronic pulmonary aspiration, 41\%; and chronic constipation, 74\%. Decreased lower esophageal sphincter tone, delayed gastric emptying, impaired esophageal motility, poor posture, recurrent seizures, scoliosis, and various medications are thought to contribute to gastroesophageal reflux disease (GERD) in neurologically impaired children. ${ }^{3}$ Respiratory symptoms, which are frequent in NIP, are also thought to aggravate GERD. ${ }^{4}$ Despite its high incidence, GERD in neurologically impaired children is difficult to recognize as the symptoms of GER are nonspecific and many patients cannot precisely express their symptoms. Therefore, diagnosis of GERD in NIP is often delayed until severe esophagitis or fatal aspiration pneumonia occurs. ${ }^{5}$ As GER is closely related with aspiration pneumonia or food refusal, this can be a large obstacle to appropriate nutritional support of NIP, which can result in a poor clinical prognosis. This is why physicians should pay attention to GER in NIP. Early suspicion and evaluation can prevent severe complications of GERD in NIP and can lead to better clinical outcomes. In this study, we analyze the degree of gastroesophageal reflux according to patient characteristics and define the risk factors for GERD in neurologically impaired children.

\section{MATERIALS AND METHODS}

\section{Study population and data collection}

Data from pediatric patients who received 24-hour esophageal pH monitoring in Severance Children's Hospital from May 2006 to March 2014 were collected. Of those cases, neurologically impaired children were selected for the study. The reasons for performing 24-hour esophageal $\mathrm{pH}$ monitoring varied, but most patients had symptoms of gastroesophageal reflux such as recurrent aspiration pneumonia, grunting after meals, frequent regurgitation, and unexplained food refusal or food-related

Correspondence to: Joon Soo Lee

Department of Pediatrics, Yonsei University College of Medicine, 50-1 Yonsei-ro, Seodaemun-gu, Seoul 03722, Korea

Tel: +82-2-2228-2050, Fax: +82-2-393-9118, E-mail: joonsl96@yuhs.ac

Received on March 18, 2016. Revised on May 13, 2016. Accepted on June 14, 2016. Published online November 14,2016

pISSN 1976-2283 eISSN 2005-1212 https://doi.org/10.5009/gnl16150

@ This is an Open Access article distributed under the terms of the Creative Commons Attribution Non-Commercial License (http://creativecommons.org/licenses/by-nc/4.0) which permits unrestricted non-commercial use, distribution, and reproduction in any medium, provided the original work is properly cited. 
irritability, or tonic posture. Patients who received upper gastrointestinal operations such as Nissen fundoplication or whose medical records were incomplete were excluded from this study. Finally, 101 patients (median age, 23.4 months) were enrolled. The results of esophageal $\mathrm{pH}$ monitoring and the clinical characteristics of the patients were analyzed. Patient selection and data collection were performed by retrospectively reviewing medical records.

\section{24-Hour esophageal pH monitoring}

To allow for an accurate diagnosis, histamine 2 receptor antagonist and proton pump inhibitor medications were discontinued at least 3 days and 7 days before examination, respectively. Prokinetic drugs were also discontinued for more than 3 days. A $\mathrm{pH}$-monitoring catheter probe was inserted through the nose to the distal esophagus. The tip of the $\mathrm{pH}$ electrode was placed on the third vertebral body above the diaphragm, as confirmed by fluoroscopy. Reflux index (\%), the percentage of the time during the investigation in which $\mathrm{pH}<4$, was used for analysis. For infants and children, more than $12 \%$ and $6 \%$ was considered pathologic reflux respectively. DeMeester score, which reflects the number of acid refluxes, number of long acid refluxes, duration of the longest acid reflux, and fraction of time that the $\mathrm{pH}$ was below 4.00, were also used for the comparison.

\section{Statistical analysis}

Reflux index was compared between groups according to diagnosis, feeding type (oral feeding, nasogastric tube feeding, or gastrostomy feeding), scoliosis status, ventriculoperitoneal shunt status, tracheostomy status, electroencephalography (EEG) results, brain magnetic resonance imaging (MRI) results and baclofen use. Correlations of reflux index and DeMeester score to disease onset age, age at examination, duration of disease, and number of antiepileptic drugs (AED) were analyzed as well. The Mann-Whitney test, Kruskal-Wallis test, and Spearman correlation test were used for the analysis. All statistical analyses were performed using SPSS software version 18.0 (SPSS Inc., Chicago, IL, USA). A p-value $<0.05$ was considered statistically significant.

\section{RESULTS}

Patients were divided according to their clinical characteristics. Distributions and descriptive statistics are given in Table 1. All patients were under the age of 18. Causes of neurologic impairment were heterogeneous and included perinatal asphyxia, genetic abnormality, cerebral hemorrhage or infarction, hypoxic brain damage, brain tumor, and infections of the central nervous system. Substantial numbers of patients had developed neurologic impairment without a definite cause. The main diagnoses of the patients were variable as well, such as LennoxGastaut syndrome, infantile spasm, cerebral palsy, mitochon- drial disease, Dravet syndrome, and so on. When we compared reflux index according to disease category, the mitochondrial disease group $(n=19)$ had a higher reflex index than did the other epilepsy group $(n=67)$ or the cerebral palsy group $(n=8)$ $(\mathrm{p}=0.009)$. The median reflux index values were $14.4,3.9$, and 3.5, respectively (Fig. 1). Patient gender, feeding method, sco-

Table 1. Patient Characteristics

\begin{tabular}{|c|c|}
\hline Variable & Value \\
\hline \multicolumn{2}{|l|}{ Categorical variable } \\
\hline \multicolumn{2}{|l|}{ Sex } \\
\hline Male & $59(58.4)$ \\
\hline Female & $42(41.6)$ \\
\hline \multicolumn{2}{|l|}{ EEG } \\
\hline Normal & $9(8.9)$ \\
\hline Abnormal & $92(91.1)$ \\
\hline \multicolumn{2}{|l|}{ Baclofen } \\
\hline With baclofen & 19 (18.8) \\
\hline Without baclofen & $82(81.2)$ \\
\hline \multicolumn{2}{|l|}{ Tracheostomy } \\
\hline With tracheostomy & $9(8.9)$ \\
\hline Without tracheostomy & $92(91.1)$ \\
\hline \multicolumn{2}{|l|}{ V-P shunt } \\
\hline With V-P shunt & $5(5.0)$ \\
\hline Without V-P shunt & $96(95.0)$ \\
\hline \multicolumn{2}{|l|}{ Scoliosis } \\
\hline With scoliosis & $36(35.5)$ \\
\hline Without scoliosis & $65(64.4)$ \\
\hline \multicolumn{2}{|l|}{ Feeding route } \\
\hline Oral & $45(44.6)$ \\
\hline Nasogastric tube & $48(47.5)$ \\
\hline Gastrostomy & $8(7.9)$ \\
\hline \multicolumn{2}{|l|}{ Main diagnosis } \\
\hline Epilepsy & $67(66.3)$ \\
\hline Mitochondrial disease & 19 (18.8) \\
\hline Cerebral palsy & $8(7.9)$ \\
\hline Others & $7(6.9)$ \\
\hline \multicolumn{2}{|l|}{ Brain MRI } \\
\hline Normal & $25(24.7)$ \\
\hline Abnormal & $76(75.2)$ \\
\hline Total & $101(100)$ \\
\hline \multicolumn{2}{|l|}{ Continuous variable } \\
\hline Age, mo & $23.4(10.5-44.1)$ \\
\hline Disease onset age, mo & $3(0-6)$ \\
\hline Duration of disease, mo & $15.4(7.35-30.75)$ \\
\hline No. of AED & $3(2-4)$ \\
\hline
\end{tabular}

Data are presented as number (\%) or median (interquartile range). EEG, electroencephalography; V-P shunt, ventriculoperitoneal shunt; MRI, magnetic resonance imaging; AED, antiepileptic drug. 


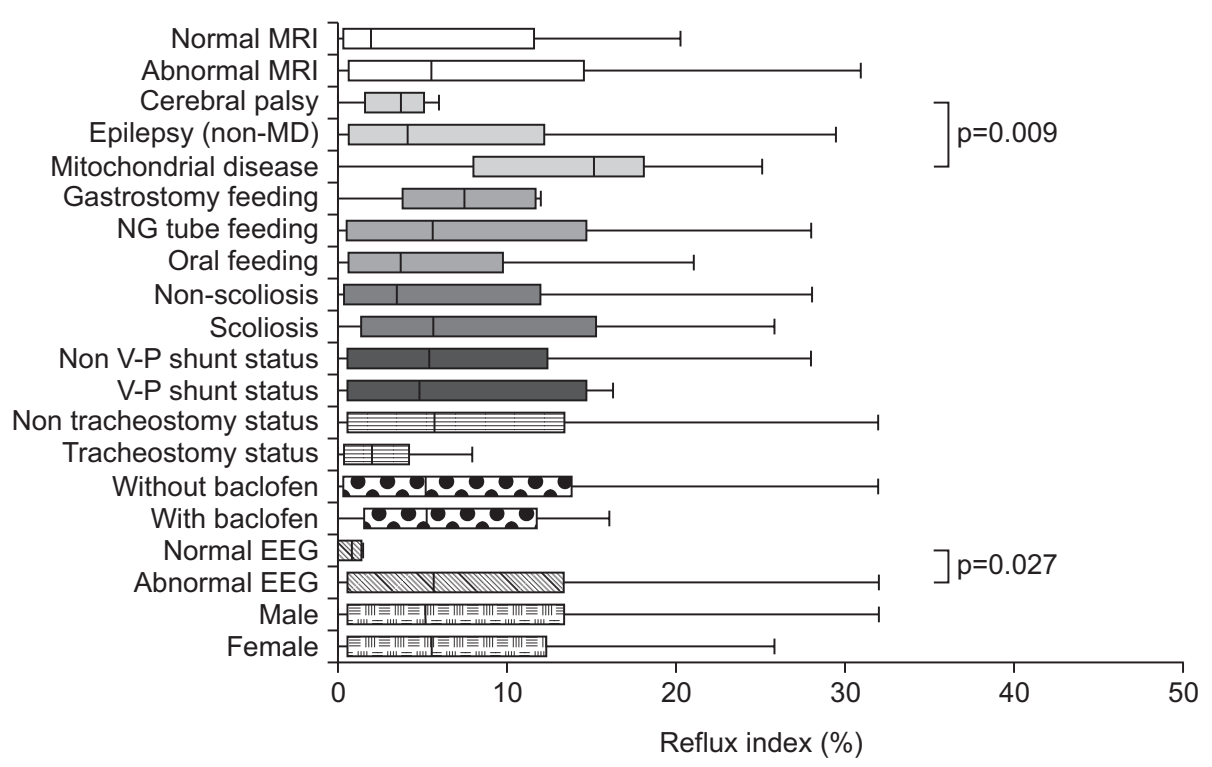

Fig. 1. Box and whisker plot of reflux index showing the reflux index distribution according to clinical condition. The box represents the interquartile range, and the line in the box indicates the median value. Differences with a pvalue of $<0.05$ are marked.

MRI, magnetic resonance imaging; MD, mitochondrial disease; $\mathrm{NG}$, nasogastric; V-P, ventriculoperitoneal shunt; EEG, electroencephalography.

Table 2. Spearman Correlation Coefficients of the Gastroesophageal Reflux Index and DeMeester Score with Continuous Variables

\begin{tabular}{|c|c|c|c|c|}
\hline & \multicolumn{2}{|c|}{ DeMeester score } & \multicolumn{2}{|c|}{ Reflux index } \\
\hline & Correlation coefficient & p-value & Correlation coefficient & p-value \\
\hline Disease onset Age & -0.389 & $<0.001$ & -0.371 & $<0.001$ \\
\hline Examination Age & -0.31 & 0.760 & -0.050 & 0.621 \\
\hline Duration of disease & 0.180 & 0.071 & 0.159 & 0.113 \\
\hline No. of AED & 0.083 & 0.409 & 0.122 & 0.224 \\
\hline
\end{tabular}

$\mathrm{AED}$, antiepileptic drug.

liosis, tracheostomy, brain MRI results and baclofen use did not lead to statistically significant differences in reflux index. Reflux index was significantly higher in the abnormal EEG group than in the normal EEG group ( $p=0.027$ ) (Fig. 1).

The age at examination, duration of the disease, and number of AED did not correlate with reflux index or DeMeester score. However, age of onset of neurological impairment was inversely correlated with DeMeester score (correlation coefficient, -0.389 ; $\mathrm{p}<0.001)$. Reflux index (\%) and disease onset age also demonstrated a similar tendency (correlation coefficient, -0.371 ; $\mathrm{p}<0.001$ ), which means that early onset of neurologic impairment can aggravate GER (Table 2).

\section{DISCUSSION}

GER refers to retrograde passage of gastric contents to the esophagus, pharynx, or oral cavity. GERs can be normal physiological events which occur frequently even in healthy individuals. ${ }^{6}$ However, severe GER can cause troublesome symptoms or complications and can impair health-related quality of life. ${ }^{7}$ This condition is called GERD. GERD is much more prevalent in NIP than in the normal population, and it can be a significant obstacle to ensuring adequate nutrition. In clinical practice, neuro- logically impaired children are often malnourished and malnutrition and GER can negatively impact one another., ${ }^{8,9}$ Therefore, both conditions should be monitored and managed attentively. Furthermore, due to a lack of effective communication, GERD is often diagnosed belatedly. GER is also closely related to respiratory problems. Recurrent pulmonary infections can be caused that give rise to poor prognoses. ${ }^{10}$ Thus, GERD can decrease a patient's quality of life both directly and indirectly. If GER can be predicted in NIP, appropriate evaluation and management measures can be taken, which will lead to better long-term outcomes. To our knowledge, this is the first study to define the clinical risk factors of GER in neurologically impaired children.

Although GER is a relatively common problem in both normal infants and NIP, the mechanisms of GER are not completely understood. Transient relaxation of the lower esophageal sphincter (LES) has been reported to be a main mechanism of GER in children. ${ }^{11,12}$ On the other hand, in NIP, absence of LES tone is thought to be the main mechanism rather than transient relaxation of the LES. ${ }^{13}$ Delayed gastric emptying and decreased antroduodenal motor function have also been suggested as mechanisms of GER in NIP. ${ }^{14,15}$ As the mechanisms of GER in NIP are complicated and poorly understood, it is difficult to predict and prevent GERD in such patients. This study was 
designed to identify the risk factors for GER in neurologically impaired children.

In this study, early-onset neurological impairment was related to more severe GER. This finding may be because early-onset neurological diseases tend to be related to congenital neurologic anomalies or genetic mutations which bring about major neurologic sequelae, and, accordingly, severe systemic complications. In a previous study, 67\% of otherwise healthy infants were found to have GER at 4 months of age. ${ }^{16}$ This is due to frequent feeding, short esophagi, the wide angle between the esophagus and stomach, and the amount of time infants spend in the supine position rather than in an upright position. ${ }^{17}$ This physiologic GER was alleviated in the course of normal growth, development, and transition to solid food. Only 5\% of otherwise healthy children have GER at 12 months of age. ${ }^{16}$ In early-onset NIP, such normal developmental progress can be halted and GER may persist throughout life. Therefore, additional attention to GERD and aspiration is necessary for patients with infantileonset neurologic impairment. Although we also hypothesized that a long duration of morbidity might affect the severity of GER, the two were not significantly associated. Disease duration does not seem to be a significant factor for progression of GER in NIP.

The clinical manifestations of neurological diseases are different according to the diagnosis. Therefore, we compared the severity of GER amongst patients with different categories of disease. As the causes and diagnoses of neurologic impairment were heterogeneous, we categorized them as mitochondrial disease, epileptic disorders other than mitochondrial disease, cerebral palsy, and others. Patients with mitochondrial disease showed a higher reflux index than did those with other diseases. Mitochondrial disease is a multisystemic disorder that is frequently associated with gastrointestinal and hepatic manifestations. ${ }^{18}$ Bhardwaj et al. ${ }^{19}$ demonstrated that children with mitochondrial disease had frequent gastrointestinal symptoms, such as abdominal pain, GER, and constipation, which are thought to be related to impaired gastric emptying and small bowel transit time, and which respond poorly to medications. This result suggests that young NIP with mitochondrial disease are at additional risk for GER. Not only neurologic impairment but also mitochondrial disease itself may contribute to this result. However, additional randomized controlled studies are required to fully elucidate this matter.

In a previous study, somewhat different characteristics were seen between nasogastric tube fed and orally fed children, and orally fed NIP were at an increased risk for aspiration. ${ }^{1}$ Feeding route and physical characteristics of food may influence upper gastrointestinal motility and gastric emptying. However, in the present study, statistical differences were not observed between the gastrostomy, nasogastric tube, and orally fed groups. As patients in the three groups tended to have different clinical conditions, confounding factors may have affected the results.
To compare objective conditions, the patients with ventriculoperitoneal shunts or tracheostomy or scoliosis were analyzed with the patients who did not have the conditions. As a result, such conditions did not statistically affect reflux activity. In the case of EEG, the normal EEG group had a lower reflux index than did the abnormal EEG group. This means that abnormal EEG can be considered a risk factor for GERD in NIP. However, this does not mean that seizure activity aggravates GERD directly, as the number of AED, which is an indicator of intractable seizures, is not correlated with reflux activity. Rather, abnormal brain function itself most likely affected reflux activity.

As many patients with neurological problems suffer from tonic posture, baclofen is sometimes used as a muscle relaxant. Because it is a GABA type B receptor agonist, baclofen is known to decrease postprandial acid reflux by reducing transient LES relaxation. ${ }^{20,21}$ Kawai et al. ${ }^{22}$ reported that administration of baclofen reduced the frequency of vomiting and acid reflux in neurologically impaired children. Although we attempted to investigate the antireflux effect of baclofen in this study, no significant differences existed between the baclofen-treated group ( $1 \mathrm{mg} / \mathrm{kg} /$ day) and the group not taking baclofen. This result may be negatively affected by spasticity itself, the reason for baclofen treatment.

This study has several limitations. First, the study population was heterogeneous. Patients with several different types of neurological impairments were included, and their clinical conditions were variable. Second, the reasons for performing 24-hour esophageal $\mathrm{pH}$ monitoring were diverse. Some patients had severe recurrent aspiration pneumonia, whereas others had mild irritability. The decision to perform the test depended on individual physicians, which may have introduced a selection bias. Although we have discontinued medications such as proton pump inhibitor or $\mathrm{H}_{2}$ blocker to exclude the medication effect, we could not completely control other medications (e.g., baclofen or AED) which might potentially affect $\mathrm{pH}$ monitoring. Generally, it is believed that combined 24-hour multichannel intraluminal impedance-pH monitoring is much more accurate and reliable method than $\mathrm{pH}$ monitoring alone, especially in monitoring the NIP. ${ }^{1}$ As we could not evaluate intraluminal impedance, this can be a main limitation of this study.

As several complex clinical factors may influence GER, it is difficult to predict the degree of GER just by using objective clinical factors in severely ill, neurologically impaired children. However, as the results of this study show, early onset neurologic impairment, mitochondrial disease, and abnormal EEG can be important risk factors for severe GER. Accordingly, early suspicion and proper evaluations of GER are needed for those patients.

\section{CONFLICTS OF INTEREST}

No potential conflict of interest relevant to this article was 
reported.

\section{REFERENCES}

1. Del Buono R, Wenzl TG, Rawat D, Thomson M. Acid and nonacid gastro-oesophageal reflux in neurologically impaired children: investigation with the multiple intraluminal impedance procedure. J Pediatr Gastroenterol Nutr 2006;43:331-335.

2. Del Giudice E, Staiano A, Capano G, et al. Gastrointestinal manifestations in children with cerebral palsy. Brain Dev 1999;21:307311.

3. Trinick R, Johnston N, Dalzell AM, McNamara PS. Reflux aspiration in children with neurodisability: a significant problem, but can we measure it? J Pediatr Surg 2012;47:291-298.

4. Aviv JE, Liu H, Parides M, Kaplan ST, Close LG. Laryngopharyngeal sensory deficits in patients with laryngopharyngeal reflux and dysphagia. Ann Otol Rhinol Laryngol 2000;109:1000-1006.

5. Gössler A, Schalamon J, Huber-Zeyringer A, Höllwarth ME. Gastroesophageal reflux and behavior in neurologically impaired children. J Pediatr Surg 2007;42:1486-1490.

6. Sherman PM, Hassall E, Fagundes-Neto U, et al. A global, evidencebased consensus on the definition of gastroesophageal reflux disease in the pediatric population. Am J Gastroenterol 2009;104:12781295.

7. Madisch A, Kulich KR, Malfertheiner P, et al. Impact of reflux disease on general and disease-related quality of life: evidence from a recent comparative methodological study in Germany. Z Gastroenterol 2003;41:1137-1143.

8. Lewis D, Khoshoo V, Pencharz PB, Golladay ES. Impact of nutritional rehabilitation on gastroesophageal reflux in neurologically impaired children. J Pediatr Surg 1994;29:167-169.

9. Marchand V, Motil KJ; NASPGHAN Committee on Nutrition. Nutrition support for neurologically impaired children: a clinical report of the North American Society for Pediatric Gastroenterology, Hepatology, and Nutrition. J Pediatr Gastroenterol Nutr 2006;43:123-135.

10. Omari T, Barnett C, Snel A, et al. Mechanism of gastroesophageal reflux in premature infants with chronic lung disease. J Pediatr Surg 1999;34:1795-1798.

11. Kawahara H, Nakajima K, Yagi M, Okuyama H, Kubota A, Okada
A. Mechanisms responsible for recurrent gastroesophageal reflux in neurologically impaired children who underwent laparoscopic Nissen fundoplication. Surg Endosc 2002;16:767-771.

12. Omari TI, Barnett CP, Benninga MA, et al. Mechanisms of gastrooesophageal reflux in preterm and term infants with reflux disease. Gut 2002;51:475-479.

13. Pensabene L, Miele E, Del Giudice E, Strisciuglio C, Staiano A. Mechanisms of gastroesophageal reflux in children with sequelae of birth asphyxia. Brain Dev 2008;30:563-571.

14. Maxson RT, Harp S, Jackson RJ, Smith SD, Wagner CW. Delayed gastric emptying in neurologically impaired children with gastroesophageal reflux: the role of pyloroplasty. J Pediatr Surg 1994;29:726-729.

15. Miki K, Harada T, Kozaiwa K, et al. Antroduodenal motor function and gastro-oesophageal reflux in neurologically impaired children, adolescents and young adults. Eur J Pediatr 1998;157:695-696.

16. Nelson SP, Chen EH, Syniar GM, Christoffel KK. Prevalence of symptoms of gastroesophageal reflux during infancy: a pediatric practice-based survey. Pediatric Practice Research Group. Arch Pediatr Adolesc Med 1997;151:569-572.

17. Bishop W. Pediatric practice gastroenterology. New York: McGraw Hill Professional, 2010.

18. Rahman S. Gastrointestinal and hepatic manifestations of mitochondrial disorders. J Inherit Metab Dis 2013;36:659-673.

19. Bhardwaj J, Wan DQ, Koenig MK, Liu Y, Hashmi SS, Rhoads JM. Impaired gastric emptying and small bowel transit in children with mitochondrial disorders. J Pediatr Gastroenterol Nutr 2012;55:194-199.

20. Zhang Q, Lehmann A, Rigda R, Dent J, Holloway RH. Control of transient lower oesophageal sphincter relaxations and reflux by the $\mathrm{GABA}(\mathrm{B})$ agonist baclofen in patients with gastro-oesophageal reflux disease. Gut 2002;50:19-24.

21. van Herwaarden MA, Samsom M, Rydholm H, Smout AJ. The effect of baclofen on gastro-oesophageal reflux, lower oesophageal sphincter function and reflux symptoms in patients with reflux disease. Aliment Pharmacol Ther 2002;16:1655-1662.

22. Kawai M, Kawahara H, Hirayama S, Yoshimura N, Ida S. Effect of baclofen on emesis and 24-hour esophageal $\mathrm{pH}$ in neurologically impaired children with gastroesophageal reflux disease. J Pediatr Gastroenterol Nutr 2004;38:317-323. 